\title{
RISKS OF CAR PARTS PRODUCTION AND SMART METROLOGY
}

\author{
Svyatoslav Yatsyshyn, Dr.Sc., Prof.; Lviv Polytechnic National University, Ukraine; \\ Andriy Slyuz, PhD Student; SE “Lvivstandartmetrology”, Ukraine; e-mail: slav.yat@gmail.com
}

\begin{abstract}
The study of the metrological risks of the car cables' production is provided in the current issue. It is proposed to develop several different sampling methods to form lots for the study. Their capabilities are evaluated according to selected criteria based on the available technology. The advantages of the dynamic method according to the possibilities of operative metrological workshops are shown. Certain advantageous factors of the method (e.g. percentage of cables to be measured; the lot's waiting time, etc.) have been identified.
\end{abstract}

Key words: Car parts; Smart metrology; Risk; Production quality; Sampling strategies.

\section{Introduction}

Sustainable development of automotive equipment has made it possible to further improve the serial production of cars in compliance with the requirements for the ecology of production. Several car equipment manufacturers have recently appeared in Ukraine. The latter include powerful cables for electrical equipment to transmit a pulse of current from the battery to the headlights and the starter, and at the time of starting the engine, current values can briefly reach hundreds of amperes. Regarding the problems with the headlights, Toyota is recalling in the United States (from 1.11.2021) 158 thousand Tundra pickups, released in 2018-2021, due to problems with the headlights, which can lead to ignition [1]. Toyota promises that its dealers if they find a defect, will fix it for free.

\section{Shortcomings}

The presence of metrologically conditioned risks has been established, while the significant electric and energy loads during the operation of cars lead to losses due to economic risks. Free elimination for customers does not mean, but only emphasizes its significant cost to the end manufacturer, which is the mentioned company. The latter should include in this cost not only the cost of troubleshooting but the cost of revocation.

\section{The Aim of the Issue}

The work aims to study the various manifestations caused by changes in the technology of production of automotive parts, metrological and economic risks, both on the sides of manufacturers, including the final manufacturer and consumers, as recommended by the revised 2017 standard [2] concerning of results processing by calibration and testing laboratories, and also the research of possibilities of hardware and software updating of smart means of production regarding the reduction of the specified risks.

\section{Consideration of Metrological and} Economic Risks in the Most Energy-Intensive Parts of the Car

In the context of the goal of the current issue, we focus on the in-depth study of statistical and metrological features of specific production technology, obtaining a general picture in terms of risk aspects, and identifying opportunities to reduce risk utilizing the smart metrology [3].

Let's consider economic and metrological risks as two groups of interrelated risks, the interdependence of which becomes particularly powerful in the energyintensive elements of the car. These include wiring parts, including cables. In the latter, the so-called weak elements and individual "points" with the maximum value of dissipated electric power are distinguished. In general, the thermodynamics of irreversible processes through its basic equation [4] considers any situation that arises in a complex technical object, estimating the dissipated power and the resulting changes in entropy. It is at these points, where the release of entropy is maximum, that a number of the complex physical, chemical and mechanical processes occur, which significantly impair the reliability of the products. Otherwise, the operation of cables made with violations of technology, explicit or implicit, increases the risks of its failure, and mainly the risks to the consumer.

\subsection{Materials approach risks}

Strict requirements for the design of the electric cable of the car are to ensure the quality, first of all, of its tips and areas of their connection to the conductor, eliminating their soldering or welding (environmentally harmful technological operation) leads to the need for mechanical crimping a series of wires forming a cable. The electrical resistance of the transition zone can be $10^{-5}-10^{-2} \Omega$, and its measurement to characterize the quality with an error of $\sim 2-5 \%$ requires a sensitivity of 
$10^{-8}-10^{-5} \Omega$. That is, Kelvin double bridges of electrical resistance become necessary [5]. However, they are quite expensive, require a significant duration for measurements, and are not able to monitor the operations for all manufactured cables: at the same time a number of them for different brands of vehicles. Therefore, such metrological means are not provided by technological schemes and are replaced, for the established and repeatedly checked technology, by equivalent, according to manufacturers, monitoring means. In such a way there were proposed the "dimensional" templates: if the template tightly fits the crimp area of the cable tip, it is considered sufficient to ensure the "normal" quality of the cable. This is how the lines for the production of modern cables operate; and this situation practically satisfies the car manufacturers, as well as the end consumers, who are the ordinary citizens. We emphasize that with the developed technology of cable production such a scheme works more or less reliably.

Since the considered technology is preceded by several previous technologies that are the technology of producing a sheet of metal of a certain thickness, the technology of making metal, the technology of extraction of raw materials, etc., the minimum deviations at any previous technological link arise the risks in subsequent links and so on up to the final consumer. Unfortunately, changes in the production of material for the manufacture of copper-alloy tips, due to depletion of ores, adjustment of metal production technology and the sheet itself for the production of tips, etc., can lead to further deviations, seemingly insignificant, but able to occur in extreme situations.

This, we believe, is the moment of starting the motor, when through the tip that compresses the conductive wire, a current of hundreds of Amperes passes. This causes it to heat up. The area of the tip adjacent to the cable is especially heated. Due to microroughness, its electrical resistance is slightly higher than the mean resistance of the conductor. There is an excessive release of electric power, which leads to its significant heating and, thus, to a further increase in power dissipated in this place. Otherwise, the process is characterized by positive feedback. In the incandescent lamp, the defective zone of the spiral is similarly overheated and it fails prematurely. This process is superimposed on the thermal release of the contact metal. As a result, the latter partially loses its shape stability, which also intensifies the hot spot.

\subsection{Economic approaches to risks}

In the world of trade, there is a well-known principle called risk sharing. In reproducing these principles, however relevant they may have been in their original context, the industry has focused on ensuring the conformity of the goods or services provided. In case of a defect, the consequences will be felt by the customer, and the consequences can be quite serious [6]. The critical component of a car whose starter has failed or a cable has overheated makes a great problem. Metrology and economics need to be smart enough to give a correct answer to the only question of interest concerning the appropriate risks.

Therefore, production practice requires daily monitoring of metrological indicators, and measuring instruments must meet the necessary specifications. Let's remember that they should be established not only based on the uncertainty budget (by determining the impact of the instrument on other uncertainty components) but also take into account the relevant acceptable risks for customers and suppliers following [7].

Based on the fact that the planned calibration is a practice that belongs to the world of legal metrology and does not always meet the real needs of industrial reality, i.e. overcoming the risks associated with every decision, we implement smart metrology. It offers a model of the usefulness of continuous monitoring in contrast to the model of arbitrary periodic one [3]. In our case, this may mean that each car should be equipped with a smart device (Kelvin bridge), the possibility of which is too early to consider. We'll focus further on the risks associated with solving the problem of transient contacts of the power part of the car cables since the presence of this problem is noticed by manufacturers in the stages preceding the car's production and use.

\subsection{Metrological approaches to risks}

The importance of an accurate and precise metrology tool was determined by empirical rules, such as the Gagemaker rule (when the accuracy tolerances of the measuring equipment should not exceed $10 \%$ of the tolerances of the parts being checked). However, with the advent of the statistical process of production management, we moved almost to the statistical determination of the probability of incorrect classification of products during the metrological study. Few parameters, such as process distribution, precision, and accuracy of the metrological instrument, measurement strategies, etc., determine the probability that a good product is classified as bad, and vice versa. Subsequently, the probability function can be converted to a number equivalent to the percentage of the incorrectly classified product. From this number, we can compute the cost of incorrect classification, which is a function of the precision, accuracy, and measurement strategy used. This value can be used to make decisions that justify new metrology capabilities, improve measurement strategies, or decide whether metrology is needed at all [8].

\section{Production of Car Parts and Its Metrological Support}

The complexity of car parts production is permanently growing. Stages of control are added, which should guarantee the high quality of products. While manufacturing car cables the necessary stages can be 
repeated. In addition, the variety of types complicates the process. Despite the need for the recommended stages, most of them may be considered unnecessary by the manufacturer. Since these are mostly not value-added steps that increase the cost of the final product, they are abandoned. The purpose of the manufacturer is to find a balance between performing the required number of metrology operations and achieving high productivity.

Let's consider approaches to optimize sampling parameters (number of samples and frequency of inspections) and develop dynamic strategies to reduce risk in the manufacture of the car cables as well as the procedure to help determine which sampling strategy is the best, focusing on metrological characteristics and risk values. Previous studies have shown that unnecessary measurements can be avoided if the sampling strategy applies only to process machines with a constant sampling rate. Procedures for changing the sampling strategy suggest calculating the coefficients for the new sampling method.

In [9], approaches to sampling optimization for different types of metrology instruments are proposed, taking into account the capabilities of metrology in terms of computing parameters, such as the performance of metrological instruments and the probability of failure. Then metrological opportunities can be better used, depending on the characteristics of monitored process equipment. Thus, more attention can be paid to identifying critical characteristics.

Regarding sampling methods, they can be classified into three main types: static, adaptive, and dynamic [10]:

- The static sampling type determines the lots to be measured at the beginning of production without any possible changes.

- The adaptive type also indicates the lots that should be measured at the beginning using the appropriate sampling rules, but with the possibility of final measurement fulfillment or not fulfillment, depending on the information collected during production.

- The dynamic sampling type is applied for measuring in real-time the best lots of products or their components, depending on the capacity of metrology and the current situation.

Deficiencies were identified for static sampling [11], where some technological tools were never used until completion, i. e. until the end of the application of the dynamic sampling for sample quality identification.

To control process machines or products, the static sampling can be assigned with a set value of the sampling factor such as " $1 / \mathrm{N}$ ", where $\mathrm{N}$ is the number of cables lots within the risk group. This means that for a ratio of $1 / 10$, one lot has to be measured from the batch of 10 produced lots. If each lot contains 10 cables, 90 cables of the same type are the at-risk area of lack of checking. Otherwise, $\mathrm{C} @ \mathrm{R}$ (cables at risk) factor is proposed as an indicator for managing the level of risk of the technological tool. It indicates the number of manufactured cables that may be compromised between two consecutive events.

In addition, it was found that the involvement of some metrological techniques does not depend on the monitored object, but depends on the capabilities of technological equipment and sampling with this equipment. In our case, such a technological tool can be considered a high-precision Kelvin double bridge for measuring the electrical resistance of the transition zones "tip - cable". This bridge limits the sampling time, as it takes $\sim 5$ minutes to determine the resistance of one cable.

In this context, it is emphasized the in-depth study of the producer's current quality control and compliance system, including the workshops to ensure the choice of the cable. The enterprises of the branch are constantly developing methods to detect deviations in the current technological processes as soon as possible while maintaining high product quality. At the same time, the study of ways to improve the reliability of processes and equipment without overloading the production system becomes a priority. To achieve the quality of technological processes and production equipment, it is recommended to perform calibrations (to control the quality of compression of the tips, dimensional calibers are used, with which each manufactured cable is checked. That is, $100 \%$ sampling is performed).

Unfortunately, the current risks due to the inadequate input control of the tips' material, lead to the occurrence and possible recurrence over time of the above-mentioned defects, which are manifested in the end-user. At the moment, we have proposed several workshops able to reduce consumer risks by increasing the risks of the producer or a group of producers. These include various additional checks or workshops aimed at replacing the operation of checking the compressed tips with a caliber. List and describe them:

- Replacement of the checking operation of the compressed tips with the help of dimensional caliber with the operation of the $3 \mathrm{D}$ vision [12]. It can be received the benefits of the applied method of rejection. However, the weakening of dimensional stability cannot be eliminated. This requires the implementation of stricter input control of the sheet material of the tips, for example by using the method of thermoelectric probe [13] powered by acoustic control.

- Addition or replacement of the checking operation of the compressed tips with the help of dimensional caliber by the measurements of the electrical resistance of the transition zone of the compressed tip. This makes it possible to determine the optimal electric property which characterizes the formation of the "hot" dot of the cable during its operation. 
Let's consider in more detail the features of dynamic sampling similar to [9]. The latter is based on its criteria for selecting the best lots for real-time measurements and depends on the current cables' production situation, without taking into account pre-defined rules that precede the measurement phase. This sampling method allows better control of metrological capacity compared to static and adaptive sampling methods.

Dynamic selection of samples has started from $[14,15]$ where a method of effective queue management for metrological research was proposed. It combines separate sampling rules into a single unique sampling solution and subsequently an algorithm capable of responding to changing producing conditions, which has improved flexibility in determining sampling rules.

The next step has consisted in the implementation of the algorithm for selecting optimal lots, relying on a set of selection rules based on assigning certain "minuses" to each of the sampling rules, so that, as a result, then using a mixed-integer linear program to select objects with a minimum sum [16]. A similar sampling program developed at AMD's plant has helped better control the length of the metrological cycle while maintaining excellent products quality [17]. To avoid excessive sampling, it was been proposed a sampling methodology [18] able to distinguish between systematic random defects using pre-established data.

The dynamic sampling method [19] which has introduced a sampling planning and execution algorithm to minimize risks, is based on a Global Sample Indicator (GSI). It has been defined to select the lots to sample and to schedule them on the measurement tools. The industrial application of dynamic sampling based on the GSI, which assigns weight to each lot entering the measurement phase and determines the lots to be measured to minimize the overall level of factory risk was performed by [20]. A simulator called S5 (Smart Sampling Scheduling and Skipping Simulator) has been implemented and validated on actual data. It shows that the risks can be strongly reduced while keeping a limited number of measures [21]. It is stated [9] that the development of dynamic sampling strategies for research requires more investment (time + money) in the development of methodology and implementation, compared to static or adaptive sampling methods.

Relying on such experience, we are working below on similar solutions with help of the mentioned methods of sampling including dynamic type, in the production of cars' cables. To obtain the final product, more than 100 technological operations are performed on cables, including preparatory operations on the reconfiguration of the technological line. In contrast to semiconductor chip manufacturing operations, where the cycle duration reaches two months, here the final cycle duration is lower than 1 work shift.

The technology must ensure that the equipment is used correctly and the cables are made following the requirements for a given type of vehicle. Labels attached to manufactured objects are used. According to the manufacturer, excessive use of metrological tools leads to production delays. The compromise between the number of metrologically justified workshops and productivity is shifted towards the latter, which causes the shortcomings identified by the consumer in cars. Only a complete assessment of the cost of recalling and repairing cars, as well as the loss of reliable brands image, can give weight to smart metrology.

\subsection{Kelvin bridge in quality control of cables} of automobile production

Measurements performed with this method are described in detail in [5]. The needed time for only one cable to be measured in a few minutes. This means that if you have the necessary metrological instrument and an experienced metrologist, with a total capacity of 1000 cables per shift, you can check $\sim 30 \%$ of the cables during the shift. Otherwise, a $30 \%$ sample is provided; the adaptive sampling method, which provides for the possibility of checking a batch of cables at the end of their manufacture, cannot be recommended.

\subsection{Probe method of studying the stability} of the material of the supplied plates for cable tips by their thermo-EMF

The probe method can be used at the stage of preparation of materials for the manufacture of cable tips by studying thermo-EMF [13]. Both the sheet from which the tips are made and the tips themselves are inspected. The material is characterized by the conformity of the reference sample, for which there are data on the cables' reliability confirmed by the consumer.

\subsection{Method of 3D dimensional vision}

The method is related to materials science and is performed automatically by a webcam when an artificial neural network is involved for image analysis. In practice, it is relevant to the above-described and widely used method of rejecting manufactured cables after the operation of crimping the body of the cable tips. It does not require much time and can be re-applied after some time with the analysis of the intensity of the initial stages of loss of stability [22].

\subsection{Development of the Sampling Rules,} Types, and Strategies

Analysis of metrological workshops data for the production of automotive parts, based on the fundamental points concerning the quality of manufactured objects, have shown the necessity to match the lot's size with the risk values (economic and metrological).

Preferably, specialized cable-production enterprises provide inspection of each cable during the manufacturing cycle with the help of a certain type of 
metrological control every 60 seconds. The metrology team consists of 2 workers using 16 autonomous metrological instruments (for such measurements as thickness, overlay, critical size, etc.), 7 instruments for detecting contaminants, particles, scratches, voids, poor seal, etc.), 12 types of tools (means for storage and protection of objects from pollution) and 4 built-in metrology tools (for electronic paper labels); total 39 items.

\subsection{Sampling and scheduling algorithms.}

Metrological approaches

It was proposed an approach to assess the correctness of sampling of the checked objects and scheduling of these samples over time in combination with technological capabilities of manufacturing as well as the metrological support of the technological line. Finally, the metrological workshops have eliminated excessive delays at the measurement stage and the stage of queues. This approach is designed primarily for the automotive industry as an industry with significant risk, including the final consumer.

The following types of scheduling of separate batches of products are considered:

- FIFO (abbreviation of the English term): First In, First Out.

The first lot that has entered the metrology zone and is intended for measurement, is checked first.

- LIFO: Last In, First Out.

The last lot that has entered the metrology zone and is intended for measurement, is measured first.

- Priority order:

Lots that reach the metrological zone are submitted for inspection following a pre-established procedure.

- Another order:

The order of the lots' studies is established arbitrarily and is not discussed in advance. It can be determined, for example, by increasing the delivery time of components or their receipt from the process equipment, which is characterized by the highest level of risk.

In addition, to applying the above scheduling rules, there are considered the possibility of fulfilling steps in terms of effective application of metrology possibilities and obtaining lower risks by changing the sampling technique [10]. In particular, this can be done using a dynamic sampling type, which implements a policy of admission to effectively manage metrology queues and which first selects the parties coming from the process equipment with its inherent higher risks.

To determine which of the methods is more acceptable, let's consider three criteria:

A) Space: evaluates the method based on the required physical space for lots of cables waiting to be measured.

B) Resources: evaluates the method based on the resources required for implementation in terms of time, money, and complexity.
C) Effectiveness: access the method based on its expected results in terms of risk and how reasonably the workshops are performed (the checked lots can minimize the risk).

The afore-mentioned methods are ranked according to the criterion from the best factor (1) to the worst one (4). Finally, the method with the lowest total value of the evaluation factor is considered the best to choose.

Table 1

\section{Evaluation of scheduling methods and sampling strategies for car parts}

\begin{tabular}{|c|c|c|c|c|}
\hline Method & Space & $\begin{array}{c}\text { Reso- } \\
\text { urces }\end{array}$ & $\begin{array}{c}\text { Effi- } \\
\text { ciency }\end{array}$ & $\begin{array}{c}\text { The factor } \\
\text { of the } \\
\text { method's } \\
\text { assessment }\end{array}$ \\
\hline FIFO & 3 & 1 & 3 & 2.33 \\
\hline LIFO & 3 & 1 & 4 & 2.67 \\
\hline Priority order & 2 & 2 & 2 & 2.00 \\
\hline $\begin{array}{c}\text { Another order } \\
\text { (dynamic } \\
\text { method) }\end{array}$ & 1 & 2 & 1 & $\begin{array}{c}1.33 \\
\text { (mean) }\end{array}$ \\
\hline $\begin{array}{l}\text { - By type of } \\
\text { cables }\end{array}$ & 1 & 3 & 1 & 1.67 \\
\hline $\begin{array}{l}- \text { By poten- } \\
\text { tially weak } \\
\text { cable compo- } \\
\text { nent, f.i. the tip }\end{array}$ & 1 & 1 & 1 & 1.00 \\
\hline $\begin{array}{l}\text { - By particular } \\
\text { team or } \\
\text { individual } \\
\text { employee }\end{array}$ & 1 & 2 & 1 & 1.33 \\
\hline
\end{tabular}

The afore-mentioned approach may lead to the introduction of a new method, different from the previous one. It is aimed at enhancing the value of metrological risk C@R due to the introduction of metrological workshops, including the minimization of the queues' duration. Such metrological operation as checking the quality of cable's tip compression by the caliber does not carry useful information in the considered situation. $\mathrm{C} @ \mathrm{R}$ value does not reduce. By replacing it with the operation of automated 3D-vision, as well as by introducing the operation of the input check of the material of the contact plate or the contact itself with help of the thermoelectric probes, it can be reduced the queues' duration.

The potentially weak cable component, that is the tips, are studied several times by dissimilar scheduling methods and sampling strategies. First, it is the input checking of tips' plate on the compliance with the standard composition. Second, the analysis of operation quality that is the $\mathrm{C} @ \mathrm{R}$ chart for a given process machine compressing the tip around the cable blank. When the current cable from the particular lot is checked with help of traditional operation applying the 
dimensional caliber, it takes minimal time and is fulfilled entirely. This time is included in the accepted previously "Process Time". Here, the traditional technology is supplemented with the stand-alone verification of the tips' platter. This does not eliminate the possibility of the weak hot spot appearance in the future arising the consumer's risk.

To reduce the probability of occurrence of these spots, it is necessary to conduct additional issues of the certain lots of manufactured cables in conditions close to natural. This means the need to study the shape stability of the tips in accelerated conditions, i.e. for the transmission of the electrical currents greater than 10 $20 \%$ of the nominal values for the operating cables. These tests should be performed on already manufactured cables, for example, by organizing a $10 \%$ sampling; again, the duration of the test and the production cycle as a whole, increases quite significantly - by $2-3 \%$. In this case, the current transmission is preceded by measurements of a) the electrical resistance of the transition zone "tip - cable"; b) verification of this area by dimensional caliber; c) relevant verification of these zone by the $3 \mathrm{D}$ vision. The same studies on samples of the same lot are repeated at the end of current tests. In the presence of significant changes that go beyond the mean values, the presence of the defects in the specified weak point of the cable is proven.

As a result, when the current lot is to be studied, the value of $\mathrm{C} @ \mathrm{R}$ increases. It increases in proportion to the number of cables waiting for these metrological workshops. Later, the measurement operation begins, which takes some time. At this point, the value of $\mathrm{C} @ \mathrm{R}$ drops to as many cables as processed. The final value of $\mathrm{C} @ \mathrm{R}$ is the difference between $\mathrm{C} @ \mathrm{R}$, reduced as a result of the measurement check, and the current value of $\mathrm{C} @ \mathrm{R}$ increased since the technological equipment produces the next batch of cables.

Thus, 2 times introduced to analyze the level of metrological risks C@R: the duration of metrological operations and the duration of waiting for the party in line for metrological activities, can better characterize the proposed policy. On average, for each metrological workshop, the time allotted for a chosen lot usually exceeds the duration of their measurement, due to the service queues' duration.

To identify opportunities to improve the quality of several units of process equipment due to the workshops, let's consider the procedure for assessing the selection and sampling of objects and the possibility of changing the sampling strategy. This approach should be repeated frequently to clarify the correctness of the used sampling model. This procedure is divided into the following main stages (Fig. 1): 1. Specification of the applied metrological instrument's performance. 2. Study of the relationship between units of technological equipment and metrological instruments. 3. Identification of possible bottlenecks in technology for its improvement due to the introduction of proposed workshops. 4. Considering the current sampling strategy, analyze the possibilities of the proposed new sampling strategy and calculate new sampling coefficients. 5. Deciding whether there are enough metrological measures to maintain the current technological support (path 5-3) or whether to change the technology and its metrological support (path 5-1)

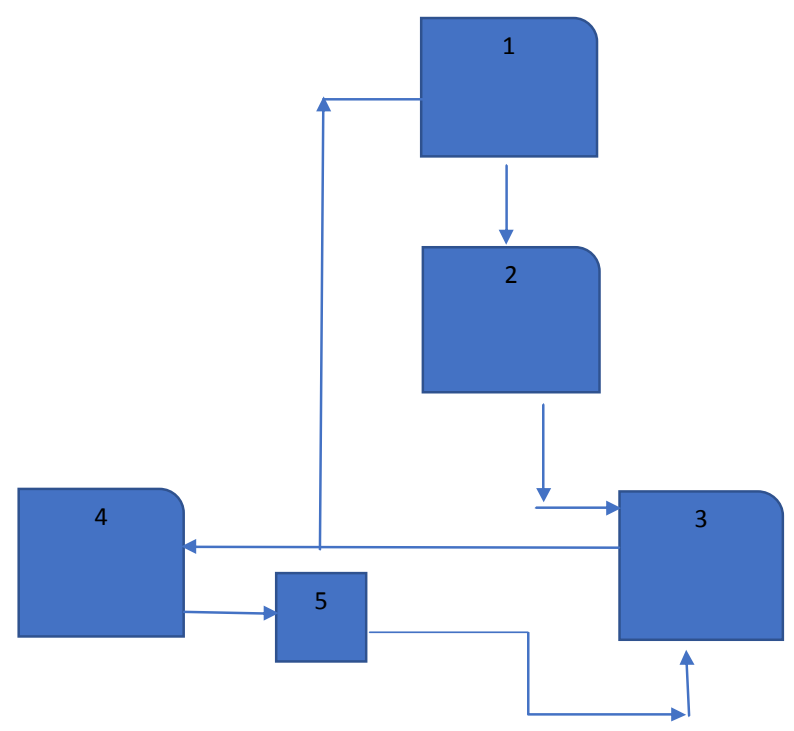

Fig. 1. Procedure for assessing the selection and determination of cables' sampling status

It can be emphasized that in this figure the $3 \mathrm{rd}$ stage is responsible for determining the correct indicators when establishing the possibility of their improvement. If the indicators are of sufficient quality, then the chosen lot remains unchanged. Otherwise, we move to the 4th stage, which introduces a new sampling strategy. Before making changes to it, is necessary to study the quality indicators to check later in which direction the process has altered due to the made changes. Here, the indicators can be the $\mathrm{C} @ \mathrm{R}$ values for the process equipment, the overall efficiency of the equipment, the duration of the cycle, the output of cables, and so on.

If it is impossible to improve the sampling methodology, for example by changing the sampling rate values or the strategy, the current scheduling system is studied aiming for its possible alteration. If the sampling strategy is maintained, then a model of checking its operability is created, with the help of which the correctness of the strategy is regularly checked. The criterion, in this case, is the expectancy of the results and at least the consistency or improvement of the $\mathrm{C} @ \mathrm{R}$ values.

In addition to $\mathrm{C} @ \mathrm{R}$ values, two other key performance indicators are subject to review. They describe whether there has been an improvement with the change in sampling strategy, namely: risk variability and changes in cycle duration. When the total risk variability for certain workshops, expressed by the 
standard deviation $\sigma$ of the obtained values, may decrease (for $\mathrm{C} @ \mathrm{R}$ values - from 78 to 48), this indicates the effectiveness of the workshops taken before.

Thus, there was carried out a detailed analysis of metrological workshops on the example of the production of cars' cables, taking into account the following: physical and design features of the components of cables, the number of units of technological equipment for their production, complexity, and skills of workshops, types of metrological instruments, lots' scheduling strategy, placement of the workshops in the production cycle, the relative number of tested cables, sampling rules and frequency, number and duration of measurements and queues, and C@R values. This makes it possible to understand how effective the metrological workshops are in key parameters, as well as how well the fulfilled decisions can improve the productivity of a particular workshop and, consequently, reduce the metrological and economic risks of manufactured cables.

\section{Consummation}

As a result of studying the combined risks of flexible production of complex and reconfigurable production of electric cars' cables, combined with several metrological workshops aimed at improving their quality, the following is established:

- There are some workshops able to improve significantly the quality of cables and reduce the risks of consumers by increasing the metrological risk of the manufacturer. To identify the potential for possible quality improvement and risk reduction, the considered workshops analysis procedure has been developed and some characteristics have been established (e.g. percentage of cables to be measured; the lot's waiting time, etc.) and the sampling methods for verification during the production cycle.

- Approaches to sampling strategy have changed and workshops based on sampling by-products and operations have been separated: product approaches should be based on variable sampling coefficients, while technological equipment approaches should be based on constant coefficients, as the latter can stand idle for some time.

- Based on the specifics of checking the cars' cables, the nomenclature of which varies from one relay to another depending on the production order, a combined sampling for studies, taking into account the capabilities of metrological instruments, was proposed. To meet the required sampling frequency, this method gives priority to sampling by product type and by lotsending operation for measurement, which is provided when the previous sampling method did not achieve the objectives.

- A relevant approach can be considered estimation of the characteristics of metrological instruments concerning the characterization of the studied cables, f.i by notification of their produced quantity.

The dynamic sampling strategy, quite effective in the electronics production, has proven its success for small-scale flexible production of car cables, as effective in lots' selection for measurements, convenient in adaption for permanent restructuring of technology (alteration of the list of cables), and on the management of metrological capabilities aiming the achievement of the reduced metrological risk and cycle time with increased productivity.

Therefore, a method for changing the sampling strategy has to be developed to identify the correct steps: to reconcile the existing sampling strategy with the capabilities of metrological instruments; to achieve an optimal risk strategy by detecting redundant metrological instruments; to identify risk mitigation concerning the next stages of production and operation of the considered cables.

\section{Gratitude}

The authors express their gratitude to the Staff of the Department of Information and Measuring Technologies of Lviv Polytechnic National University.

\section{Conflict of interest}

The authors state that there are no financial or other potential conflicts regarding this work.

\section{References}

[1] Toyota Recalls Tundra Pickups for Headlights That Catch Fire. Consumer Reports, Sept. 2021 [Online]. Available: https://www.consumerreports.org/car-recalls-defects/toyotatundra-pickup-recall-for-headlights-that-catch-fire-a1208906316/

[2] ISO/IEC 17025:2017. General requirements for the competence of testing and calibration laboratories [Online]. Available: https://www.iso.org/obp/ui/\#iso:std:iso-iec:17025:ed3:v1:en

[3] Cyber-Physical Systems and Metrology 4.0. Coeditors S. Yatsyshyn, B.Stadnyk, IFSA Publishing, Barcelona, 2021. [Online]. Available: https://www.sensorsportal.com/ HTML/BOOKSTORE/Cyber-Physical_Systems_and_Metrology 4_0.htm

[4] Ya. Demirel, V.Gerbaud, Nonequilibrium Thermodynamics, in Chapter 1. Fundamentals of Equilibrium Thermodynamics, Elsevier, 2019. [Online]. Available: https://www. elsevier.com/books/nonequilibrium-

thermodynamics/demirel/978-0-444-64112-0

[5] L. Jones, A. Chin, Electronic Instruments and Measurements, Prentice-Hall,1991. [Online]. Available:

[6] J.-M. Pou, L.Leblond, "Smart Metrology: From the metrology of instrumentation to the metrology of decisions", Art. 01007, Jan. 2017 Conf.: 18th Int. Congress of Metrology, [Online]. Available:https://doi.org/10.1051/metrology /201701007

[7] ISO/IEC Guide 98-4:2012. Uncertainty of measurement - Part 4: Role of measurement uncertainty in conformity assessment. [Online]. Available: https://www.iso. org/ru/standard/50465.html 
[8] S. Kudva, R. Potter, "Cost analysis and risk assessment for metrology applications", in Proc. Vol. 1673, Integrated Circuit Metrology, Inspection, and Process Control VI; Event: Microlithography '92, USA, 1992. [Online]. Available: https://ui.adsabs.harvard.edu/abs/1992SPIE.1673....2K/abstract

[9] J. Perez, Risk minimization through metrology in semiconductor manufacturing. Université de Lyon. HAL Archives, English. ffNNT : 2017LYSEM022 , 2017 [Online]. Available: https://tel.archives-ouvertes.fr/tel-02878704/document

[10] Ju. Nduhura-Munga, G. Rodriguez-Verjan, S. Dauzere-Peres, C. Yugma, Ph. Vialletelle, J. Pinaton. "A literature review on sampling techniques in semiconductor manufacturing". IEEE Trans. on Semicond. Manufact., Vol. 26, Iss.2, pp.88-195, 2013. [Online]. Available: https://www. researchgate.net/publication/260638406_A_Literature_Review on_Sampling_Techniques_in_Semiconductor_Manufacturing

[11] Ju. Nduhura-Munga. Implementing and optimizing dynamic control plans in semiconductor manufact., $\mathrm{PhD}$ thesis, Ecole Nat. Supérieure des Mines de Saint-Etienne, Gardanne, France, 2012.

[12] 3D-vision on Windows 10. NDIVIA. [Online]. Available: https:/www.nvidia.com/en-us/geforce/forums/3dvision/41/286802/3d-vision-on-windows-10/

[13] PCE Instruments. [Online]. Available: Defectoscope PCE-FD 20. https://www.pce-instruments.com/english/ measuring-instruments/test-meters/defectoscopekat 162404.htm.

[14] M.-G. Shin, J.-H. Lee, “An Auto Metrology Sampling Method Considering Quality and Productivity for Semiconductor Manufacturing Process", Trans. of The Korean Inst. of Electr. Eng., Vol.61, Is.9, pp.1330-1335, 2012 [Online]. Available: http:/www.koreascience.or.kr/article/JAKO201225067516719.page.

[15] K. Wu, Modeling for Semiconductor Industry Dynamics, Mass.Inst. of Techn., 2008. [Online]. Available: https://studylib.net/doc/

11277284/modeling--the-semiconductor--industry-dy-kailiang--wu.

[16] P. Espadinha-Cruz, R.Godina, E.M.G.Rodrigues. A Review of Data Mining Applications in Semiconductor Manufacturing, Processes, 9, 305, 38 p.,. 2021, [Online]. Available: https://doi.org/10.3390/pr9020305 https://www.mdpi. com/jour.

[17] G. Verjan, "Smart Sampling for Risk Reduction in Semicinductor Manufacturing", HAL Archives, [Online]. Available: https://tel.archives-ouvertes.fr/tel-01126975.

[18] Ya. Kaga, Yo. Sato, Ya. Yamada, Yu. Yamazaki, M. Aoki, R. Harukawa, E. Chang, "Integrated defect sampling method by using design attribute for high sensitivity inspection in $45 \mathrm{~nm}$ production environment", In Semiconductor Manufacturing, 2008 Int. Symp., pp.379-381. IEEE, 2008.

[19] A. Lima, V. Borodin, S. Dauzère-Pérès, P. Vialletelle, "A sampling-based approach for managing lot release in time constraint tunnels in semiconductor manufacturing", Int. Journ. of Prod. Res., Vol.59, Iss.3, pp.860-884, 2021. [Online]. Available: https://scholar.google.fr/citations?view_ $\mathrm{op}=$ view_citation $\& \mathrm{hl}=$ fr\&user=T7mDFAEAAAA.

[20] S. Housseman, S. Dauzère-Pérès, G. RodriguezVerjan, J. Pinaton, "Smart dynamic sampling for wafer at risk reduction in semiconductor manufacturing", In Aut. Sc. and Eng. (CASE), 2014 IEEE Int. Conf., pp.780-785. IEEE, 2014.

a. P. Vialletelle, S. Dauzère-pérès, C. Yugma, J. Pinaton, "A Smart Sampling Scheduling and Skipping Simulator and its evaluation on real data sets", in Proc. 2011 Winter Simulation Conf. (WSC), 2011 [Online]. Available: https://www.academia.edu/17594430/A_Smart_Sampling_Sched uling_and_Skipping_Simu-lator_and_its_evaluation on real data sets

[21] Vision and Learning Lab., Seoul National University, 2021 [Online]. Available: https://cse.snu.ac.kr/en/lab/ vision-and-learning-lab 